\title{
多孔钲钛矿型氧还原催化剂在柔性铝空气电池中的应用研究
}

\author{
税子怡 ${ }^{a}$ 何娜娜 ${ }^{a}$ 陈黎 ${ }^{a}$ 赵炜 $*, a$ 陈曦 $*, a, b$ \\ $\left({ }^{a}\right.$ 西北大学化工学院 西安 710069) \\ ( ${ }^{b}$ 哥伦比亚大学地球与环境学院能源环境先进材料研究中心 纽约 NY10027)
}

\begin{abstract}
摘要 钙钛矿型催化材料作为贵金属的低成本替代物受到广泛关注，其用作氧还原反应催化剂具有非常可观活性和稳 定性. 目前相关工作主要集中在使用钻钛矿制备技术影响元素组成、形态、表面积和结构控制等方面. 对于普遍存在 的钙钛矿 $\left(\mathrm{ABO}_{3}\right)$ 材料而言，制备过程㷽烧温度较高导致合成材料比表面积通常较小，限制了其在非均相催化反应中活 性的提升. 本文借助无机模板 $\mathrm{SiO}_{2}$ 制备具有高活性的大比表面的钲钛矿型催化材料 $\mathrm{La}_{0.7} \mathrm{Sr}_{0.3} \mathrm{MnO}_{3}$ (LSMO). 测试结果 表明, 模板法 LSMO 具有非常可观的比表面积 $\left(31.1825 \mathrm{~m}^{2} \cdot \mathrm{g}^{-1}\right)$, 用在柔性铝空气电池中放电电压相比于无模板法和溶 胶凝胶法可分别提高 $8.2 \%$ 和 $24.5 \%$, 且在大电流放电下性能衰退明显减缓. 在电池变形状态下，输出电压可稳定在 $1.38 \mathrm{~V}$ 以上. 不仅为燃料电池商业化提供了解决方案, 同时为未来可变性电源发展提供了新方向. 关键词 钻钛矿氧化物; 比表面积; 氧还原反应; 催化活性; 柔性铝空气电池
\end{abstract}

\section{Porous Perovskite towards Oxygen Reduction Reaction in Flexible Aluminum-Air Battery}

\author{
Shui, Ziyi ${ }^{a} \quad \mathrm{He}, \mathrm{Nana}^{a} \quad \mathrm{Chen} \mathrm{Li}^{a} \quad$ Zhao, Wei*,a $\quad$ Chen, $\mathrm{Xi}^{*}, a, b$ \\ $\left({ }^{a}\right.$ Northwest University, School of Chemical Engineering, Xi'an 710069) \\ ( ${ }^{b}$ Columbia University, Department of Earth and Environmental Engineering, New York NY10027)
}

\begin{abstract}
Perovskite-type catalytic materials have received wide attention as high-performance and low-cost alternatives to precious metal catalysts on the market at present, which have much considerable activity and stability as catalysts for oxygen reduction reactions. Current efforts are mainly focused on the use of perovskite make-up and preparation techniques to influence elemental composition, morphology, surface area, and structural control. For a typical perovskite oxide $\left(\mathrm{ABO}_{3}\right)$, due to the high calcination temperature in the preparation process, the perovskite material usually has a small specific surface area, which limits the increase of activity in heterogeneous catalytic reactions. In this paper, the perovskite $\mathrm{La}_{0.7} \mathrm{Sr}_{0.3} \mathrm{MnO}_{3}(\mathrm{LSMO})$ material with large specific surface and high catalytic activity is prepared by means of the $\mathrm{SiO}_{2}$ template. The physicochemical properties of the synthesized materials are characterized by scanning electron microscope (SEM), energy dispersed X-ray spectroscopy (EDS), X-ray diffraction (XRD) and BET. The catalytic activity of LSMO as an oxygen reduction reaction (ORR) catalyst is measured by a rotating disk test system. After that, the catalyst material is applied to a flexible aluminum-air battery and its discharge behavior and flexibility is studied and tested. The test results show that the LSMO prepared by template method has a large specific surface area $\left(31.1825 \mathrm{~m}^{2} \cdot \mathrm{g}^{-1}\right)$, and pore volume $\left(0.161113 \mathrm{~cm}^{3} \cdot \mathrm{g}^{-1}\right)$, and it also shows higher electrocatalytic activity in the electrochemical test system. When it is used in aluminum-air batteries, the activity of 3D porous LSMO is significantly better than that of sheet and bulk LSMO. The aluminum-air battery assembled by LSMO prepared by the template method has a higher discharge voltage (up to $1.46 \mathrm{~V}$ ) at a constant current. Compared to the template-free method and the sol-gel method, the discharge voltage in flexible aluminum- air battery can be increased by $8.2 \%$ and $24.5 \%$, respectively, and the performance degradation is significantly slowed during high-current discharge. The specific capacity and energy density of the battery are up to $1048.6 \mathrm{~mA} \cdot \mathrm{h} \bullet \mathrm{g}^{-1}$ and $1020.6 \mathrm{~mW} \cdot \mathrm{h} \cdot \mathrm{g}^{-1}$, respectively. When the battery is in a deformed state, its output voltage can be stabilized above $1.38 \mathrm{~V}$. Once released, the voltage can be immediately restored to over $99 \%$ of the initial value. This paper not only provides a solution for the commercialization of fuel cell, but also provides a new direction for the future development of variable power supply.
\end{abstract}

Keywords perovskite oxide; specific surface area; oxygen reduction reaction; catalytic activity; flexible aluminum-air battery

\footnotetext{
* E-mail: zhaowei3313@nwu.edu.cn; xichen863@hotmail.com

Received March 14, 2020; published May 18, 2020.

Project supported by the National Natural Science Foundation of China (No. 11872302), the Natural Science Basic Research Program of Shaanxi Province (No. 2019JQ-431) and the Shaanxi Provincial Department of Education Natural Science Special Project (No. 20JK0927).

项目受国家自然科学基金(No. 11872302)、陕西省自然科学基础研究计划(No. 2019JQ-431)和陕西省教育厅自然科学专项(No. 20JK0927)资助.
} 


\section{1 引言}

化石能源日益枯竭, 环境问题日益突出, 目前急需 寻找一种高效且环保的新型能源转化系统以满足国家 供应需求 ${ }^{[1]}$. 金属-空气电池成为电化学电源领域中非 常具有竞争力的化学电源之一 ${ }^{[2]}$. 铝空气电池由于具有 较高的理论比容量、重量轻、环境友好且成本低廉被视 为下一代非常有前景的储能设备 ${ }^{[3,4]}$. 然而刚性电池结 构限制其在柔性电子领域的应用, 因此在一定程度上可 弯曲、扭曲、卷起或拉伸且仍能保持其原有功能 ${ }^{[5]}$ 的可 变形电源, 是铝空气电池新的发展方向. 铝空气电池主 要由铝阳极、电解质和空气阴极组成 ${ }^{[6]}$. 空气电极是影 响电池性能最主要的因素, 其中, 氧还原的反应(ORR) 动力学缓慢且需要发生在非常高的超电势下 ${ }^{[7]}$, 因此探 寻合适的电催化剂是提高 ORR 效率和降低超电势的关 键. 贵金属催化剂及其合金 ${ }^{[8 \sim 11]}$ 、过渡金属氧化物催化

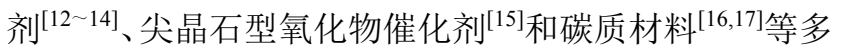
种电催化材料已被用作阴极催化剂, 其中 Pt 或 Pt 基合 金催化性能优异，但容易中毒失活和高成本阻碍了其应 用. 最新研究表明钙铁矿氧化物 $\left(\mathrm{ABO}_{3}\right.$, 其中 $\mathrm{A}$ 是稀土 元素, $\mathrm{B}$ 是过渡金属元素)可作为替代贵金属的最佳氧还 原反应催化剂之一 ${ }^{[18 ~ 21], ~} \mathrm{LaMnO}_{3}$ 是燃料电池和金属空 气电池中最常用的电催化剂. 据报道 ${ }^{[22]}$ 用 $\mathrm{Ca}$ 或 $\mathrm{Sr}$ (LCM 或 LSM) 部分取代 $\mathrm{La}$ 可以进一步提高 $\mathrm{LaMnO}_{3}$ 的 催化活性, 这可能与钙钛矿结构的改变以及 $\mathrm{Mn}^{3}$ 和 $\mathrm{Mn}^{4+}$ 之间的锰价态的操纵有关 ${ }^{[18,19,23,24]}$. 为了进一步提 高催化剂的活性, $\mathrm{Liu}$ 等 ${ }^{[25]}$ 证实 $\mathrm{LaMnO}_{3}$ 的多孔结构有利 于传质并改善了 ORR 过程. 但由于煅烧温度高, 钙钛矿 型材料的比表面积通常较小, 因此如何制备具有多孔结 构的大比表面积钙铁矿催化剂成为研究的新方向. Lee 等 ${ }^{[26]}$ 通过表面活性剂模板法制备了具有分级介孔结构 的立方相 $\mathrm{LaMnO}_{3}$, 最大比表面积可达到 $23 \mathrm{~m}^{2} \cdot \mathrm{g}^{-1}$. 有 机模板广泛用于多孔材料的制备程且容易去除, 但其在 相对较低的温度易燃烧, 制备过程不安全是致命的弱 点.

本工作通过 Stöber-Frink 方法 ${ }^{[27]}$ 合成了纳米尺度的 $\mathrm{SiO}_{2}$ 模板, 由于其高温下稳定性而被用于制备纳米多孔 钙钛矿 $\mathrm{La}_{0.7} \mathrm{Sr}_{0.3} \mathrm{MnO}_{3}$ (LSMO). 同时在相同条件下不使 用模板和使用常见的柠檬酸络合法分别制备无模板法 LSMO 和溶胶凝胶法 LSMO 作为参照物, 具体合成步骤 如图 1 所示. 通过旋转圆盘测试系统检测 LSMO 在 ORR 过程中的电催化活性. 采用原位生长方法将 LSMO 材料 长于碳布表面制成柔性空气电极, 以铝箔作为阳极材料 配合丙烯酰胺水凝胶电解质膜 ${ }^{[28]}$, 采用夹层设计 ${ }^{[29]}$ 组 装成柔性铝空气电池, 进行全电池放电行为研究和短期 受力下的柔性测试.

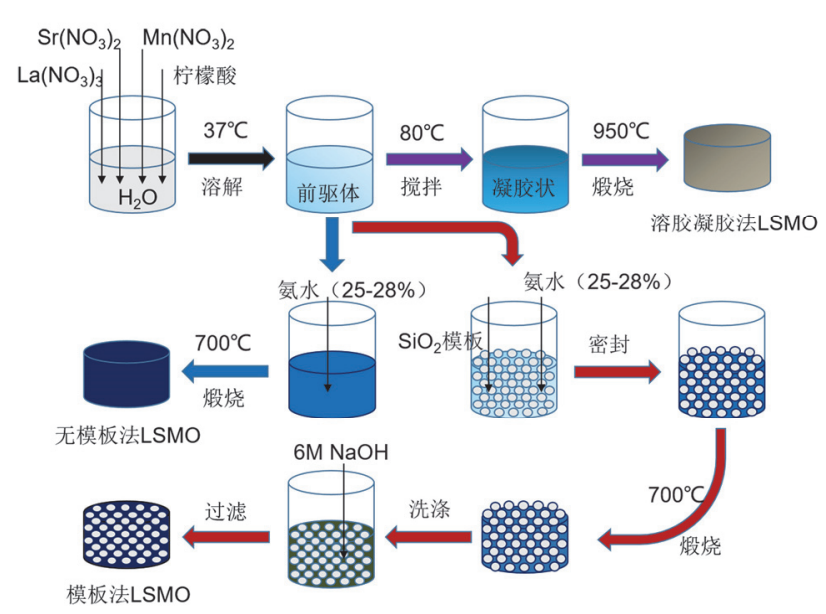

图 1 三种 LSMO 样品的合成路线

Figure 1 Synthesis route of three LSMO samples

\section{2 结果与讨论}

\section{1 球形 $\mathrm{SiO}_{2}$ 模板形貌观察}

球形 $\mathrm{SiO}_{2}$ 模板的场发射扫描电镜(FESEM)如图 2a 所示, 其中球体的大小均匀 $(330 \mathrm{~nm})$, 且在空间内分散 排列. 实际上, 它是由面心立方(FCC)结构的单分散胶 体 $\mathrm{SiO}_{2}$ 颗粒的三维自组装形成. 从 $\mathrm{EDS}$ 光谱(图 2b)中 可以看出, 样品中仅存在 $\mathrm{Si}$ 和 $\mathrm{O}, \mathrm{Si}$ 原子与 $\mathrm{O}$ 原子的比 例为 $1: 2$. (a)

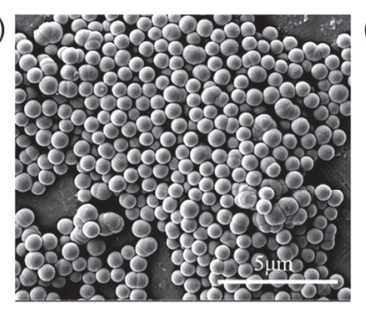

(c)

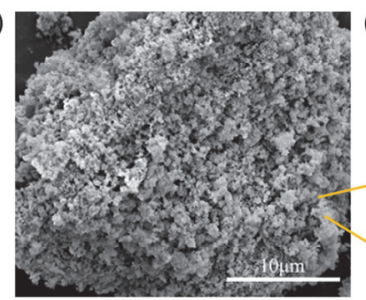

(e)

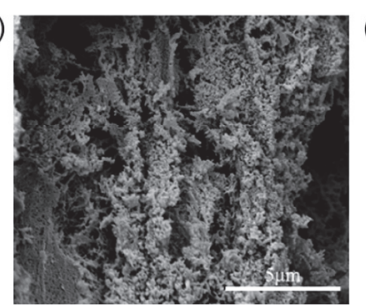

(b)

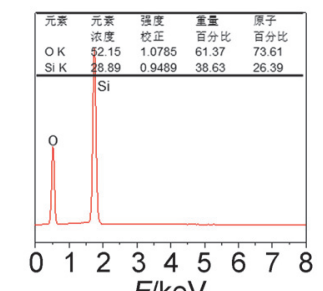

$\mathrm{E} / \mathrm{keV}$
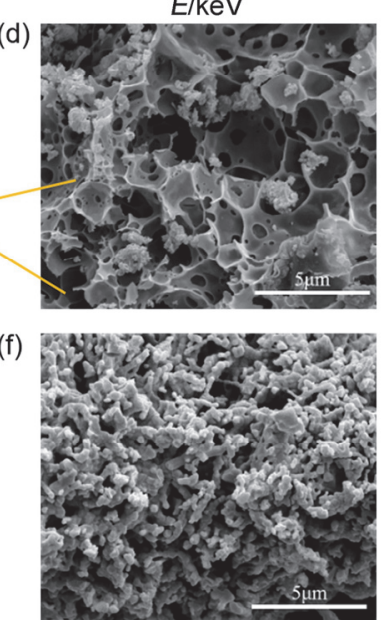

图 $2 \mathrm{SiO}_{2}$ 模板的 (a) SEM 图像和(b) EDS 光谱、(c, d)模板法、(e)无模 板法和(f)溶胶凝胶法 LSMO 的 SEM 图像

Figure 2 (a) $\mathrm{SEM}$ image and (b) EDS spectrum of $\mathrm{SiO}_{2}$ template, $\mathrm{SEM}$ image of (c, d) template method LSMO, (e) template-free method LSMO and (f) sol-gel method LSMO 


\section{2 钙钛矿催化剂材料表征}

使用场发射扫描电子显微镜测定了三种 LSMO 样 品的微观形貌结构, 从图 2 中可以清楚地观察到, 与使 用无模板法和溶胶凝胶法制备的 $\mathrm{La}_{0.7} \mathrm{Sr}_{0.3} \mathrm{MnO}_{3}$ 相比, 在使用模板法制备的 $\mathrm{La}_{0.7} \mathrm{Sr}_{0.3} \mathrm{MnO}_{3}$ 材料的表面和体相 观察到了更多的孔洞，表明采用模板制备的 LSMO催化 剂在保留了材料本身的孔道结构的同时增加了模板去 除后形成的多孔结构. 当其被用作空气电极的催化剂 时, 参与反应的液-气-固的三相界面(TPI)极大地影响 催化性能, 并且催化活性随着 TPI 增多而增加. 图 2d 所 示的模板法合成的 $\mathrm{La}_{0.7} \mathrm{Sr}_{0.3} \mathrm{MnO}_{3}$ 的显微图像证实了我 们的猜想, 除去 $\mathrm{SiO}_{2}$ 微球模板后, 钲钛矿材料仍保留了 多孔结构, 其归因于连续的 $\mathrm{SiO}_{2}$ 颗粒在空间中形成了 连续的 3D 球形多孔结构. 由于暴露在 SEM 图像中的多 孔结构未呈现出明显的分布规律, 因此无法直接对孔的 结构和尺寸进一步分析和测量. $\mathrm{SiO}_{2}$ 微球模板之间紧密 接触, 在形成的多孔钙钛矿氧化物之间出现了微小的连 接节, 这不仅会大大提高 TPI, 同时提高了传质水平, 暴露出更多的氧化活性位点.

模板法、无模板法和溶胶凝胶法合成的催化剂样品 的 $X$ 射线衍射图如图 3 所示. $X$ 射线衍射结果确认了这 项工作中合成的三种 LSMO 样品的钙钛矿相的形成 (PDF: 089-8097). XRD 数据表明 $\mathrm{SiO}_{2}$ 模板的机械引入 (搅拌) 与化学移除(添加 $\mathrm{NaOH}$ ) 不影响多孔 $\mathrm{La}_{0.7} \mathrm{Sr}_{0.3}-$ $\mathrm{MnO}_{3}$ 的晶体结构.

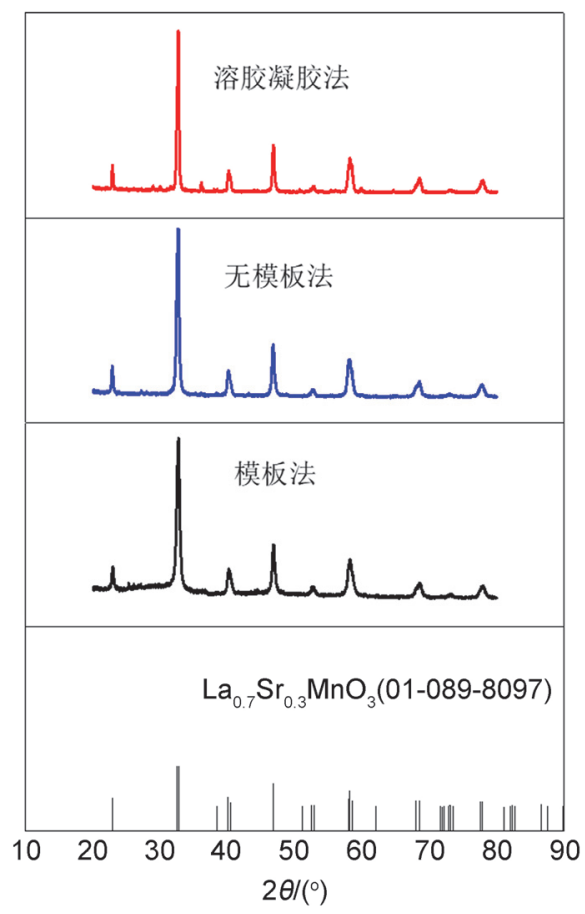

图 3 三种 LSMO 样品的 XRD 图

Figure 3 XRD patterns of three LSMO samples

为进一步研究催化剂内部孔结构, 使用 $\mathrm{N}_{2}$ 吸收一解 吸实验研究孔的尺寸和分布(图 4, 表 1). $\mathrm{SiO}_{2}$ 模板的引
入有利于钙钛矿材料多孔结构的形成，与无模板法 $\left(15.3771 \mathrm{~m}^{2} \cdot \mathrm{g}^{-1}\right)$ 和溶胶凝胶法 $\left(5.0825 \mathrm{~m}^{2} \cdot \mathrm{g}^{-1}\right)$ 制备的 $\mathrm{La}_{0.7} \mathrm{Sr}_{0.3} \mathrm{MnO}_{3}$ 相比, 借助无机模板形成具有多孔结构 的 $\mathrm{La}_{0.7} \mathrm{Sr}_{0.3} \mathrm{MnO}_{3}$ 具有明显增大的比表面积，其比表面 积为 $31.1825 \mathrm{~m}^{2} \cdot \mathrm{g}^{-1}$, 分别为无模板法比表面积的 2 倍 和溶胶凝胶法比表面积 6 倍左右. 与 Lee 的表面活性剂 模板方法 ${ }^{[23]}$ 相比，用 $\mathrm{SiO}_{2}$ 模板制备的 3D 多孔 $\mathrm{LaMnO}_{3}$ 的比表面积几乎是其 1.5 倍 $\left(23 \mathrm{~m}^{2} \cdot \mathrm{g}^{-1}\right)$, 其原因主要是 有机模板在一定的温度下 (大多数有机模板在 $500{ }^{\circ} \mathrm{C}$ 以 下分解)会分解从而导致多孔结构塌陷. 但 $\mathrm{SiO}_{2}$ 模板的 热稳定性可以解决此问题, 并且在 $\mathrm{NaOH}$ 溶液蚀刻后保 留了 $3 \mathrm{D}$ 多孔结构.

表 $1 \mathrm{~N}_{2}$ 吸附-脱附试验及相应的孔径分布

Table $1 \mathrm{~N}_{2}$ adsorption-desorption test and corresponding pore size distribution

\begin{tabular}{lccc}
\hline & 比表面积 $/\left(\mathrm{m}^{2} \cdot \mathrm{g}^{-1}\right)$ & 孔体积 $/\left(\mathrm{cm}^{3} \cdot \mathrm{g}^{-1}\right)$ & 孔径 $/ \mathrm{nm}$ \\
\hline 模板法 LSMO & 31.1825 & 0.161113 & 20.6671 \\
无模板法 LSMO & 15.3771 & 0.080547 & 29.1404 \\
溶胶凝胶法 LSMO & 5.0825 & 0.011301 & 9.4025 \\
\hline
\end{tabular}

\section{3 电催化活性分析}

通过循环伏安法(CV)和线性扫描伏安法(LSV)表征 采用模板法、无模板法、溶胶凝胶法制备的 $\mathrm{La}_{0.7} \mathrm{Sr}_{0.3}$ $\mathrm{MnO}_{3}$ 材料在 ORR 的电催化活性. 图 $5 \mathrm{a}$ 为三种 LSMO 催化剂的循环伏安法 $(\mathrm{CV})$ 曲线(样品均在 $\mathrm{O}_{2}$ 饱和溶液中 进行测定). 三种 LSMO 样品的还原峰出现在 $-0.3 \sim$ $-0.42 \mathrm{~V}$ 之间. 其中, 模板法制备的 LSMO 在 $-0.4 \mathrm{~V}$ 处 出现最大正还原峰, 最大峰值电流密度为 $1.65 \mathrm{~mA}$ $\mathrm{cm}^{-2}$. 该结果进一步证实了模板法 $\mathrm{La}_{0.7} \mathrm{Sr}_{0.3} \mathrm{MnO}_{3}$ 具有 比无模板法和溶胶凝胶法更优异的电催化活性. 除此之 外, 将纯相钙钛矿氧化物材料单独用作电催化剂时, 钙 钛矿相对较差的导电性导致其表现出较弱的氧还原活 性, 适当掺入导电炭黑的复合材料则表现出优异的氧还 原电催化活性和稳定性 ${ }^{[30]}$. 图 5b 为 LSMO 和导电炭黑 以不同比例掺杂用作催化材料时的 $\mathrm{CV}$ 曲线, 经过一系 列测定和对比证实当 $\mathrm{LSMO} / \mathrm{C}$ 以 $1: 1$ 的质量比混合时, 催化剂油墨具有最强的还原峰, 其峰值电流密度为 1.65 $\mathrm{mA} \cdot \mathrm{cm}^{-2}$. 随后, 进一步用 $\mathrm{CV}$ 研究不同催化剂负载量 时模板法 LSMO 的电催化活性(图 5c). 结果显示在 LSMO 和导电炭黑质量比为 $1: 1$ 时, 随着催化剂负载 量的增加, 其峰值电流密度依次增大. 为了进一步探究 ORR 反应机理, 采用旋转圆盘电极对反应动力学及反 应产物的稳定性进行表征. 图 $5 \mathrm{~d}$ 为在 $\mathrm{O}_{2}$ 饱和的 $\mathrm{KOH}$ 溶液 $\left(0.1 \mathrm{~mol} \cdot \mathrm{L}^{-1}\right)$ 中以 $1600 \mathrm{r} \cdot \mathrm{min}^{-1}$ 的转速进行 $\mathrm{ORR}$ 期 间在 LSMO 催化剂上收集的盘电流密度 $\left(i_{\mathrm{d}}\right)$. 图 $5 \mathrm{e}$ 为在 $\mathrm{O}_{2}$ 饱和的 $0.1 \mathrm{~mol} / \mathrm{L} \mathrm{KOH}$ 中通过分别在 100, 200, 400, 900 和 $1600 \mathrm{r} \cdot \mathrm{min}^{-1}$ 转速下在旋转圆盘电极测试系统中 使用 LSV 评估模板法 LSMO 的 ORR 活性. 在各种电极 电位下相应的 Koutecky-Levich 图 $\left(j^{-1}\right.$ 对 $\left.\omega^{-1 / 2}\right)$ 显示出良 

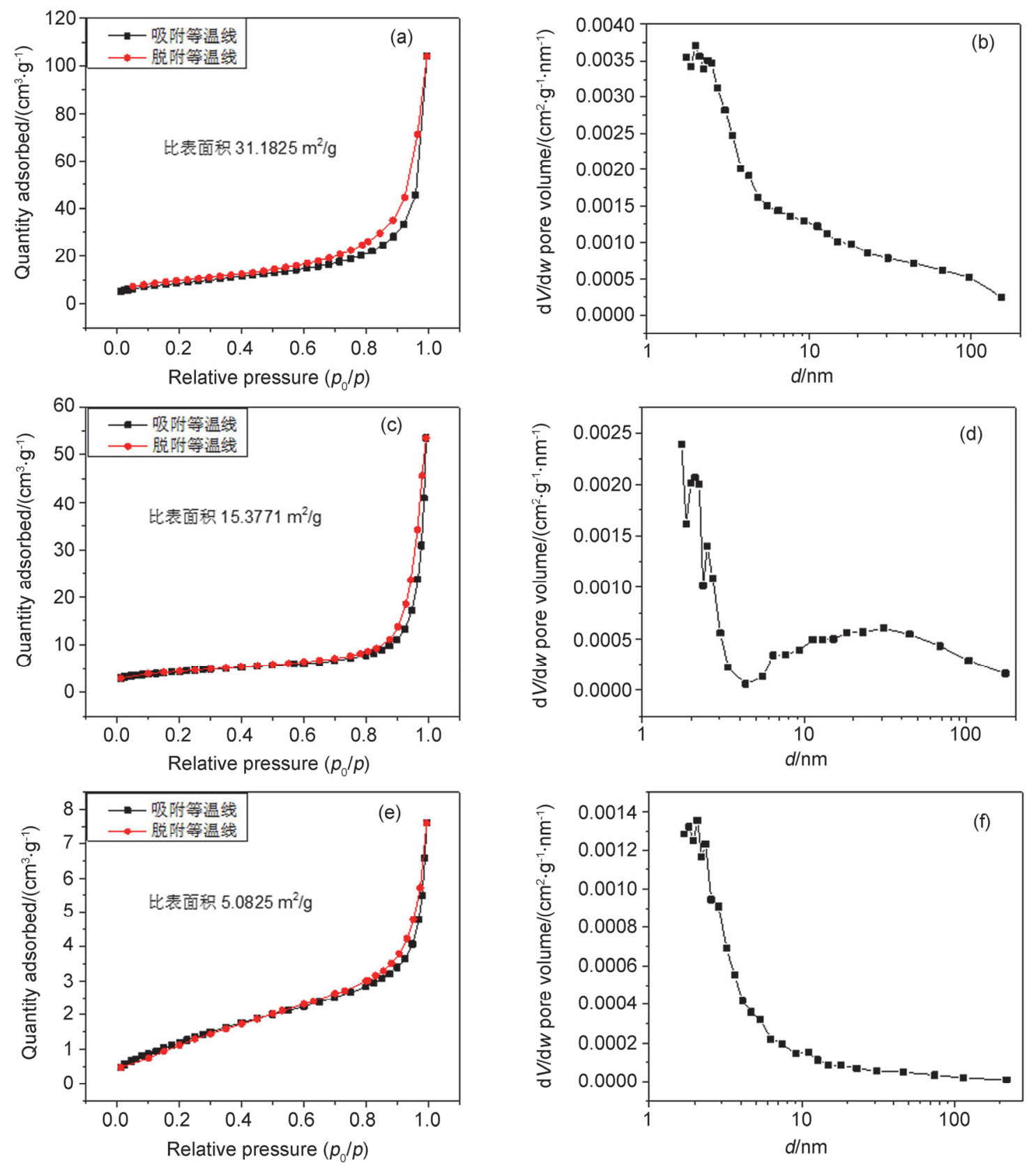

图 $4 \mathrm{~N}_{2}$ 吸附-脱附等温线和相应的孔径分布

Figure $4 \mathrm{~N}_{2}$ adsorption-desorption test and corresponding pore size distribution

$(\mathrm{a}, \mathrm{b})$ template method LSMO; $(\mathrm{c}, \mathrm{d})$ template-free method LSMO; $(\mathrm{e}, \mathrm{f})$ sol-gel method LSMO

好的线性, 表明 ORR 是相对于 $\mathrm{O}_{2}$ 溶解浓度的一级反应 动力学的典型模型. 可以在 Koutecky-Levich 方程的基 础上分析动力学参数: $N$ 是评估 ORR 中催化剂性能的重 要参数, 从 $K-L$ 曲线的斜率可以看出, 相对于 -0.8 至 $-0.4 \mathrm{~V}$ 的电位范围, $N$ 接近 4(分别为 3.72 至 3.24)(vs. $\mathrm{Ag} / \mathrm{AgCl}$ ), 表明 ORR大部分遵循四电子转移途径, 二电 子转移的副反应被有效控制, 期间产生的过氧化氢量非 常有限, 燃料利用率明显提升. 通过旋转圆盘伏安法对 电子转移动力学进行了详细研究, 结果表明, 对于模板 法和无模板法 LSMO, 在 $-0.8 \sim-0.4 \mathrm{~V}$ (vs. $\mathrm{Ag} / \mathrm{AgCl}$ )
的电势范围内, 其具有类似于 $\mathrm{Pt} / \mathrm{C}\left(N \approx 4 \mathrm{e}^{-}\right)$催化 $\mathrm{ORR}$ 的简便四电子转移动力学, 而溶胶凝胶法 LSMO 催化剂 在 $-0.8 \sim-0.4 \mathrm{~V}$ 电势范围内的电子转移数在 $2 \sim 4$ 之 间变化(图 5f).

\section{4 全电池测试}

在催化剂制备过程中采用原位生长的方法将其生 长于碳布表面制备柔性空气电极. 图 6 可看出, 不使用 任何粘结剂, 通过直接生长将催化剂颗粒负载碳布上具 有非常可观的负载量, 同时催化剂颗粒在碳布表面分布 较为均匀. 使用此方法避免使用粘结剂, 不仅能够实现 

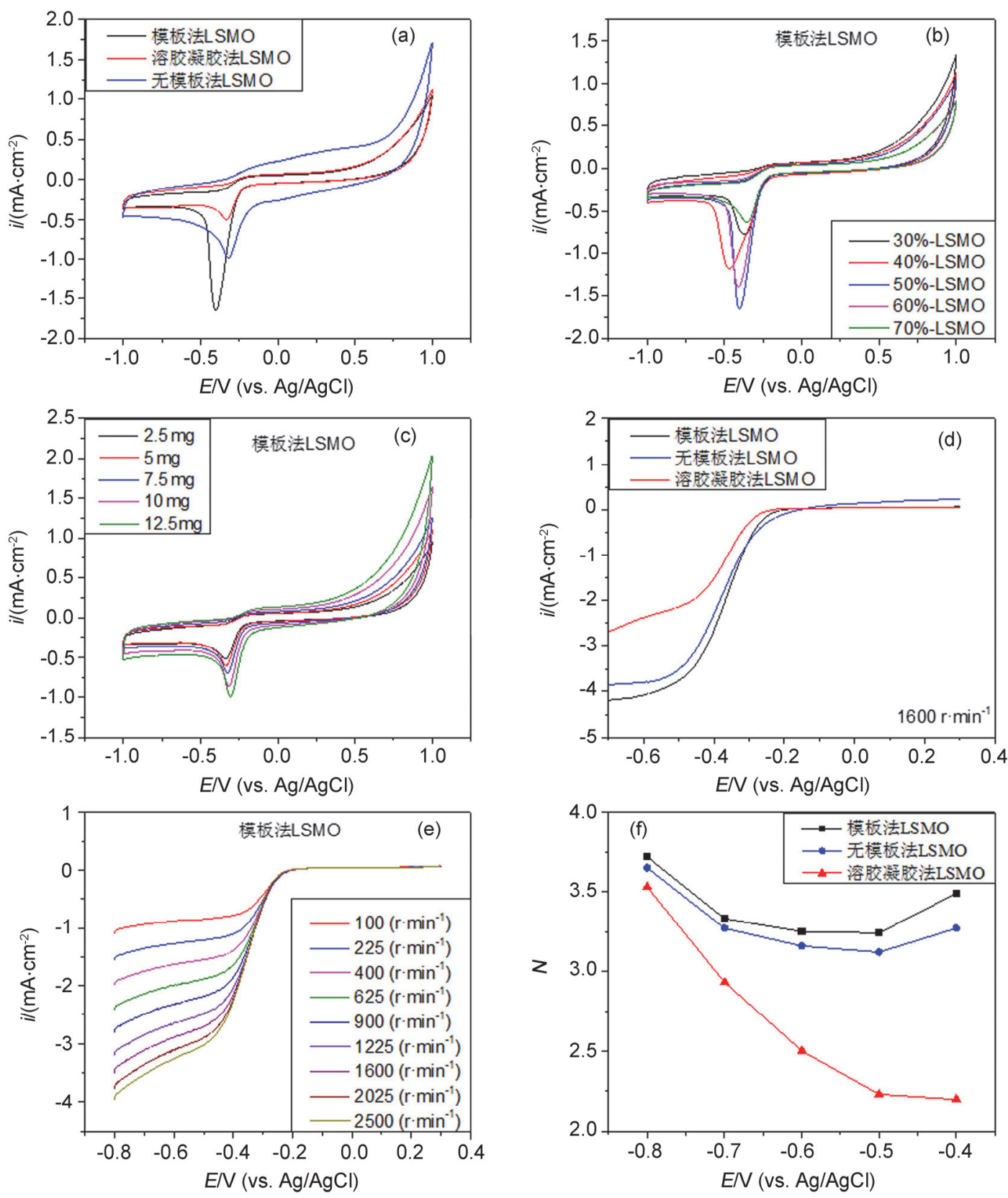

图 5 LSMO 样品电催化活性

Figure 5 Electrocatalytic activity of LSMO samples

(a) CV curves for three samples, (b) CV curves for different carbon additions, (c) CV curves for different catalyst loadings, (d) LSV for three samples at $1600 \mathrm{r} \cdot$ $\min ^{-1}$, (e) LSV of template method LSMO at different speeds, (f) number of electron transfer of three samples at various potentials

(a)
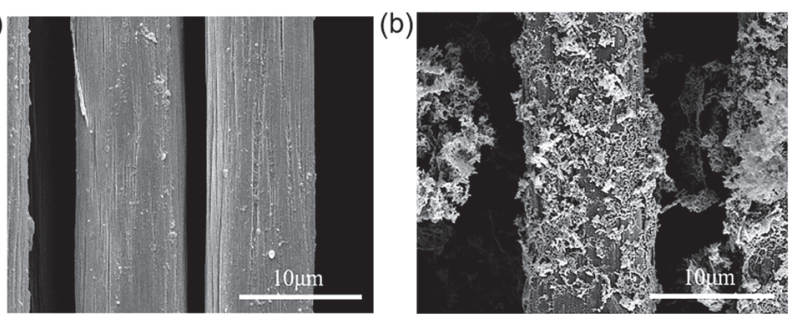

图 6 催化剂生长(a)前和(b)后碳布的 SEM

Figure 6 SEM of carbon cloth (a) before and (b) after catalyst growth
较为理想活性物质负载，而且实验过程简便容易操作， 其局限性在于负载量难以定量控制。总而言之，将活性 物质原位生长在柔性基底表面的方法为制备无粘结剂 柔性电极提供了参考意义.

为了评估三种阴极材料在铝空气电池中的放电性 能，使用铝箔作为阳极材料，丙烯酰胺聚合制成水凝胶 电解质膜，将其采用夹层设计组装成柔性铝空气电池. 在环境空气中, 室温下采用 $1,5,10$ 和 $20 \mathrm{~mA} \cdot \mathrm{cm}^{-2}$ 放电 电流分别对其进行放电并记录放电过程. 采用三种不同 
方法制成的 LSMO 电池放电曲线如图 7a 所示. 所有电 池的电压均随着放电电流的增加而降低. 使用 $\mathrm{SiO}_{2}$ 模 板构建的 3D 多孔结构极大地促进了放电电压和电池稳 定性的提升. 与溶胶凝胶法和无模板法的 LSMO 相比, 由模板法制备的 LSMO 组装的全铝空气电池在 $1,5,10$ 和 $20 \mathrm{~mA} \cdot \mathrm{cm}^{-2}$ 的电流下分别具有更高的放电电压(分别 为 $1.46,1.42,1.37$ 和 $1.32 \mathrm{~V})$, 相比于无模板法和溶胶凝 胶法分别提升 $8.2 \%$ 和 $24.5 \%$, 且在大电流放电稳定性明 显增强. 低电流密度下三种样品放电性能较为接近, 增 加电流密度时电池性能表现出明显的区别. 使用 3D 多 孔 LSMO 的电池性能明显优于片状和块状 LSMO, 主要
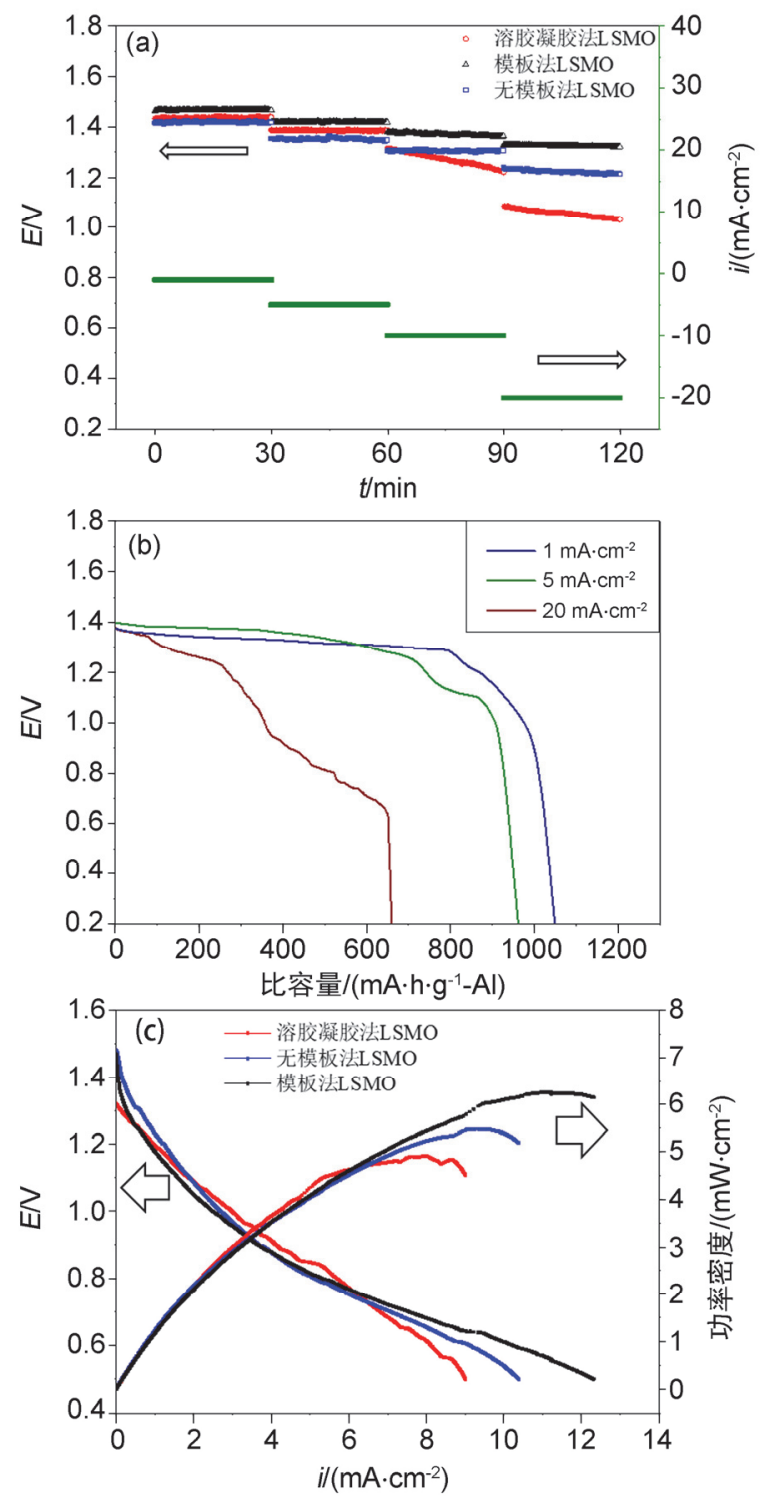

图 7 (a)三种样品恒流放电性能对比、(b)模板法制备的 LSMO 组装 的全铝空气电池在不同电流密度下恒流放电曲线和(c)极化和功率密 度曲线

Figure 7 (a) Constant current discharge performance comparison of three samples, (b) constant current discharge curve at different current densities of aluminum-air battery assembled by LSMO prepared by the template method and (c) polarization and power density curves
归因于 $\mathrm{SiO}_{2}$ 模板的引入增加了钲钛矿氧化物的孔隙率, 从而导致其比表面积和孔体积明显增大，这与上述 BET 结果一致. 增强的孔隙率促使参与反应的三相界面 (TPI)和催化活性位点显著增加. 三相界面(TPI)的增加 有助于扩大微孔间的传质，促使反应过程稳定性增强， 催化活性位点的增加则使得电池放电性能相较于其他 两者明显提升. 放电电流也会影响电池的比容量和能量 密度. 在 $1 \sim 20 \mathrm{~mA} \cdot \mathrm{cm}^{-2}$ 的恒定电流下测试了使用模板 法制备的 LSMO 材料组装的柔性铝空气电池的放电性 能(图 7b). 当电流密度小于 $5 \mathrm{~mA} \cdot \mathrm{cm}^{-2}$ 时, 放电电压保 持稳定, 放电过程结束时急剧下降表明 $\mathrm{Al}$ 的量已耗尽 并且电解质过量. 在 $20 \mathrm{~mA} \cdot \mathrm{cm}^{-2}$ 区域之上, 由于电解 质中的离子扩散不足，电压随时间下降剧烈. 如表 2 所 示，放电过程中仅考虑消耗的 $\mathrm{Al}$ 的重量，当电池以 1 $\mathrm{mA} \cdot \mathrm{cm}^{-2}$ 放电时, 最高比容量可达 $1048.6 \mathrm{~mA} \cdot \mathrm{h} \cdot \mathrm{g}^{-1}-\mathrm{Al}$, 此时能量密度达到峰值为 $1020.6 \mathrm{~mW} \bullet \mathrm{h} \cdot \mathrm{g}^{-1}-\mathrm{Al}$. 相当于 掺氮石墨烯用作铝空气电池氧还原反应电催化剂 ${ }^{[31]}$ 时 比容量的 4 倍左右, 具有与使用 $\mathrm{La}_{2} \mathrm{O}_{3}, \mathrm{SrO}$ 和 $\mathrm{MnO}_{2}$ 混 合催化剂组装而成的铝空气电池 ${ }^{[32]}$ 相当的比容量和能 量密度.

表 2 电池不同电流密度放电比容量和能量密度汇总

Table 2 Summary of different current density discharge specific capacity and energy density of batteries

\begin{tabular}{cccc}
\hline 放电电流 $/\left(\mathrm{mA} \cdot \mathrm{cm}^{-2}\right)$ 电压 $/ \mathrm{V}$ 比容量 $/\left(\mathrm{mA} \cdot \mathrm{h} \cdot \mathrm{g}^{-1}\right)$ 能量密度 $/\left(\mathrm{mW} \cdot \mathrm{h} \cdot \mathrm{g}^{-1}\right)$ \\
\hline 1 & 1.38 & 1048.6 & 1020.6 \\
5 & 1.41 & 961.9 & 934.7 \\
20 & 1.27 & 659.7 & 688.6 \\
\hline
\end{tabular}

通过电池的极化曲线和功率密度曲线进一步探讨 铝空气电池的电化学性能. 对比三种方法制备的 LSMO，发现模板法 LSMO 在相同电压下具有最高的极 化电流密度. 相比于无模板法和溶胶凝胶法 LSMO, 极 化电流密度在 $0.5 \mathrm{~V}$ 时从 9 和 $10.4 \mathrm{mg} \cdot \mathrm{cm}^{-2}$ 升高至 12.3 $\mathrm{mA} \cdot \mathrm{cm}^{-2}$ (图 7c), 模板法 LSMO 材料用于铝空气电池时 表现出明显增加的电流密度, 与其比表面积的增加呈现 出相同的规律, 可解释为构建 3D 多孔大表面积 LSMO 材料导致引入的额外活性位点促进电池性能的提升. 功 率密度和催化剂含量也出现了类似的趋势. 因此, 且模 板法 LSMO 具有最大的功率密度, 其最大功率密度对应 于 $11 \mathrm{~mA} \cdot \mathrm{cm}^{-2}$ 的电流密度, 为 $6.27 \mathrm{~mW} \cdot \mathrm{cm}^{-2}$.

将铝空气电池制成柔性，即意味着电池可以在一定 范围内变形而不损坏结构, 而输出电压在特定放电电流 下保持基本恒定. 图 8 显示了铝空气电池在不同弯曲程 度时的放电曲线. 当弯曲到 $30^{\circ}$ 时, 柔性铝空气电池的 输出电压也保持在 1.41 以上, 即使在弯曲 $90^{\circ}$ 的高度变 形下电压依然保持在 $1.38 \mathrm{~V}$ 以上. 释放后，电压仍可恢 复到 $99 \%$ 以上. 考虑到电池的安全性, 短期受力下的稳 定放电对于实际应用至关重要. 铝空气电池的柔性使其 非常适合于为便携式电子设备供电. 


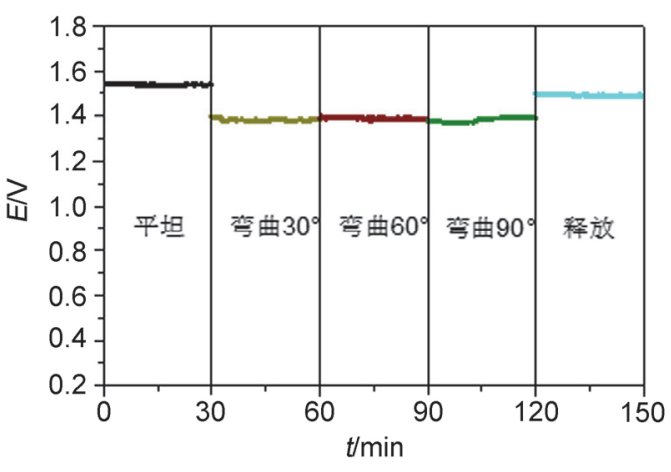

图 8 铝空气电池在不同受力状态下放电曲线 $\left(1 \mathrm{mV} \cdot \mathrm{s}^{-1}\right)$

Figure 8 Discharge curve of aluminum air battery under different stress conditions $\left(1 \mathrm{mV} \cdot \mathrm{s}^{-1}\right)$

\section{3 结论}

通过模板法、无模板法、溶胶凝胶法合成了三种 LSMO 钙钛矿型催化剂, 然后通过 SEM, EDS, XRD, BET 对合成的 $\mathrm{SiO}_{2}$ 模板和 LSMO 的物理化学性质进行 研究. 之后通过循环伏安法和放电试验研究了三种样品 的电化学性能并对组装好的全电池进行柔性测试. 结果 表明借助 $\mathrm{SiO}_{2}$ 模板制备的 LSMO 具有较高的比表面积 $\left(31.1825 \mathrm{~m}^{2} \cdot \mathrm{g}^{-1}\right)$ 和孔体积 $\left(0.161113 \mathrm{~cm}^{3} \cdot \mathrm{g}^{-1}\right)$, 在电化学 测试体系内也表现出更高的电催化活性. 将其用于铝空 气电池时, 3D 多孔 LSMO 的活性明显优于片状和块状 LSMO. 由模板法制备的 LSMO 组装的全铝空气电池在 恒定电流下分别具有更高的放电电压(最高可达 $1.46 \mathrm{~V}$ ). 当电池以 $1 \mathrm{~mA} \cdot \mathrm{cm}^{-2}$ 放电时, 最高比容量可达 1048.6 $\mathrm{mA} \cdot \mathrm{h} \cdot \mathrm{g}^{-1}$, 此时能量密度达到峰值为 $1020.6 \mathrm{~mW} \cdot \mathrm{h} \cdot \mathrm{g}^{-1}$, 当电池处于不同弯曲程度时其输出电压也保持在 1.38 $\mathrm{V}$ 以上. 一旦释放后, 电压可恢复到初始的 $99 \%$ 以上. 通过 $\mathrm{SiO}_{2}$ 模板合成的 $3 \mathrm{D}$ 多孔钙铁矿用于柔性铝空气电 池时非常适合于为便携式电子设备供电.

\section{4 实验部分}

\section{1 紧密堆积 $\mathrm{SiO}_{2}$ 模板的合成}

采用 Stöber-Frink 方法 ${ }^{[27]}$ 合成单分散 $\mathrm{SiO}_{2}$ 模板, 具 体步骤包括: 首先将正硅酸乙酯 TEOS $(99 \mathrm{~mL})$ 、去离子 水 $(81 \mathrm{~mL})$ 、无水乙醇 $(270 \mathrm{~mL})$ 和氨水 $(24 \mathrm{~mL})$ 依次倒入 烧杯, 使用磁力搅拌器密封搅拌 $48 \mathrm{~h}$, 将所得样品置于 通风梪室温下静置直至上层清液完全蒸发, 之后将其置 于真空干燥箱直至 $\mathrm{SiO}_{2}$ 模板完全烘干.

\section{2 钙钛矿 $\mathrm{La}_{0.7} \mathrm{Sr}_{0.3} \mathrm{MnO}_{3}(\mathrm{LSMO})$ 的制备}

将 $\mathrm{La}\left(\mathrm{NO}_{3}\right)_{3}(0.035 \mathrm{~mol}), \mathrm{Sr}\left(\mathrm{NO}_{3}\right)_{2}(0.015 \mathrm{~mol})$, $\mathrm{Mn}\left(\mathrm{NO}_{3}\right)_{2}(0.05 \mathrm{~mol})$, 柠檬酸 $(0.15 \mathrm{~mol})$ 与蒸馏水 $(20$ $\mathrm{mL})$ 混合搅拌 $1 \mathrm{~h}$. 取 $20 \mathrm{~mL}$ 前驱体混合溶液缓慢渗入 $\mathrm{SiO}_{2}$ 模板中, 加入过量的氨水并用塑料薄膜密封静置 $12 \mathrm{~h}$, 将所得样品在烘箱中烘干. 对烘干的前驱体粉末 进行研磨并以 $1{ }^{\circ} \mathrm{C} \cdot \mathrm{min}^{-1}$ 的升温速率从室温升至
$700{ }^{\circ} \mathrm{C}$ 并保持 $2 \mathrm{~h}$. 随后在恒定机械搅拌下将 $\mathrm{NaOH}(6$ $\mathrm{mol} \cdot \mathrm{L}^{-1}$ )溶液加入到所得样品中搅拌 $48 \mathrm{~h}$, 使用去离子 水 $\left(60{ }^{\circ} \mathrm{C}\right)$ 重复超声波洗涤和离心三次, 分离钻钛矿粉 末并在 $80{ }^{\circ} \mathrm{C}$ 干燥. 在相同条件下不使用模板制备另一 样品作为对照实验. 通过常见的溶胶凝胶 (柠檬酸络合) 方法制备另一对照物. 将金属硝酸盐前驱体以 $\mathrm{La}_{0.7} \mathrm{Sr}_{0.3}-$ $\mathrm{MnO}_{3}$ 的化学计量比溶解在具有等物质的量柠檬酸的去 离子水中, 然后将其保持在 $80{ }^{\circ} \mathrm{C}$ 水浴中搅拌直至凝胶 完成. 再将凝胶置于烘箱内进行干燥. 最后, 以 $1{ }^{\circ} \mathrm{C}$. $\min ^{-1}$ 的升温速率从室温至 $700{ }^{\circ} \mathrm{C}$ 下煅烧 $2 \mathrm{~h}$ 获得 LSMO 粉末.

\section{3 材料表征}

通过 X 射线衍射(XRD, D/MAX-3D，日本)用 $\mathrm{Cu} K \alpha$ 辐射 $(\lambda=0.15418 \mathrm{~nm})$ 分析制备的催化剂的晶体结构; 通 过场发射扫描电子显微镜(FESEM(Carl Zeiss)SIGMA 500) 测定 $\mathrm{SiO}_{2}$ 模板和催化剂的微观结构; 在 Quantachrome Instruments NOVA2200e 比表面积分析仪 下测试比表面积; 通过 $\mathrm{X}$ 射线光电子能谱仪(XPS, Thermo SCIENTIFIC ESCALAB 250Xi)评估电催化剂表 面的电子结构并进行元素组成及表面分析，其中 $\mathrm{Al} \mathrm{K \alpha}$ 辐射具有 $0.2 \mathrm{eV}$ 能量增量, $100 \mathrm{eV}$ 通过能量.

\section{4 电化学测试}

所有电化学评估均在 RST 电化学工作站进行. 旋 转圆盘测试系统(PINE)用于测试催化剂的氧化还原活 性. 测试系统为三电极体系, 包含工作电极(玻碳圆盘 电极, $5 \mathrm{~mm})$ 、参比电极 $(\mathrm{Ag} / \mathrm{AgCl})$ 和辅助电极 $(\mathrm{Pt}$ 丝). 通 过将催化剂粉末和导电炭黑(共 $10 \mathrm{mg}$ )分散在 $1 \mathrm{~mL}$ 异丙 醇中, 并加入质量分数为 $5 \%$ 的 Nafion 试剂 $(40 \mu \mathrm{L})$ 作为 聚合物粘合剂来制备催化剂油墨. 在超声处理约 $2 \mathrm{~h}$ 后, 取 $5 \mu \mathrm{L}$ 悬浮液滴到玻碳盘电极上进行测试. 使用旋转 圆盘测试系统对获得样品的循环伏安扫描 $(\mathrm{CV})$ 测试和 具有不同的旋转速率 $(100,400,900,1600$ 和 $2500 \mathrm{r} \cdot$ $\mathrm{min}^{-1}$ )的线性扫描伏安(LSV)测试. 之后将催化剂生长 于碳布表面作为空气电极, 以铝馢作为阳极材料并配合 丙烯酰胺水凝胶电解质膜, 组装成夹层式柔性铝空气电 池(图 9)并对其进行全电池放电行为研究和短期变形

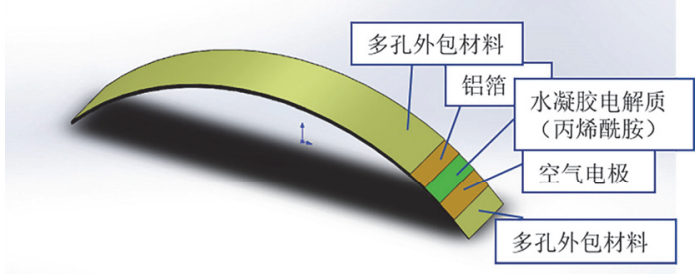

图 9 铝空气电池的结构

Figure 9 Structure of the aluminum-air battery 
下柔性测试, 获得在空气环境下的恒电流放电曲线和极 化曲线.

\section{References}

[1] Song, M. J.; Shin, M. W. Appl. Surf. Sci. 2014, 320, 435.

[2] Arora, P.; Zhang, Z. J. Chem. Rev. 2004, 104, 4419

[3] Xu, Y.; Zhao, Y.; Ren, J.; Zhang, Y.; Peng, H. Angew. Chem., Int. Ed. 2016, 55, 7979.

[4] Que, Y.; Qi, M.; Shi, P. Chin. Battery Ind. 2019, 23, 147. (阙奕鹏, 齐敏杰，史鹏飞，电池工业, 2019, 23, 147.)

[5] Xie, K.; Wei, B. Adv. Mater. 2014, 26, 3592.

[6] Cheng, F.; Chen, J. Chem. Soc. Rev. 2012, 41, 2172.

[7] Cheng, F.; Chen, J. Acta Chim. Sinica 2013, 71, 473. (程方益, 陈 军, 化学学报, 2013, 71, 473.)

[8] Hong, Q.; Lu, H. Sci. Rep. 2017, 7, 3378.

[9] Li, Y. C.; Xu, Z. C.; Gasteiger, H. A.; Chen, S.; Hamad-Schifferli, K.; Yang, S.-H. J. Am. Chem. Soc. 2010, 132, 12170.

[10] Meng, H.; Shen, P. K. Electrochem. Commun. 2006, 8, 588.

[11] Hao, J.; Liu, Y.; Li, W.; Li, J. Mater. Rev. 2019, 33, 127. (郝佳瑜, 刘易斯，李文章，李洁，材料导报, 2019, 33, 127.)

[12] Jin, Q.; Pei, L.; Hu, Y.; Du, J.; Han, X.; Cheng, F.; Chen, J. Acta Chim. Sinica 2014, 72, 920. (靳琪, 裴龙凯, 胡宇翔, 杜婧, 韩晓 鹏, 程方益, 陈军, 化学学报, 2014, 72, 920.)

[13] Wang, D.; Chen, X.; Evans, D.-G.; Yang, W. Nanoscale 2013, 5, 5312 .

[14] Wang, Y.; Wei, Z. J. Electrochem. 2018, 24, 427. (王尧, 魏子栋, 电 化学, 2018, 24, 427.)

[15] Yin, W.; Shen, Y.; Zou, F.; Hu, X.; Chi, B.; Huang, Y. ACS Appl. Mater. Interfaces 2015, 7, 4947.

[16] Liu, M.; Zhang, R.; Chen, W. Chem. Rev. 2014, 114, 5117.

[17] Liu, L.; Yuan, Z.; Qiu, C.; Liu, J. Solid State Ionics 2013, 241, 25.
[18] Wang, Y.; Zhang, L.; Hu, T. Acta Chim. Sinica 2015, 73, 316. (王瀛, 张丽敏, 胡天军, 化学学报, 2015, 73, 316.)

[19] Miyazaki, K.; Kawakita, K.; Abe, T.; Fukutsuka, T.; Kojima, K.; Ogumi, Z. J. Mater. Chem. 2011, 21, 1913.

[20] Lin, S.; Xu, S.-F.; Wang, J.-D.; Xie, C.-S.; Yuan, A.-H.; Han, G.-F.; Zhang, L.; Zhang, L.-M.; Li, Y.; Yan, Z.-M. Acta Chim. Sinica 2005 63,385 . (林生岭，徐绍芬，王俊德，谢春生，袁爱华，韩光范，张 琳, 张黎明, 李燕, 严寽明, 化学学报, 2005, 63, 385.)

[21] Takeguchi, T.; Yamanaka, T.; Takahashi, H.; Watanabe, H.; Kuroki, T.; Nakanishi, H.; Orikasa, Y.; Uchimoto, Y.; Takano, H.; Ohguri, N.; Matsuda, M.; Murota, T.; Uosaki, K.; Ueda, W. J. Am. Chem. Soc. 2013, 135, 11125.

[22] Xue, Y.; Miao, H.; Sun, S.; Wang, Q.; Li, S.; Liu, Z. J. Power Sources 2017, 342, 192.

[23] Stoerzinger, K. A.; Lü, W.; Li, C.; Ariando; Venkatesan, T.; Yang, S.-H. J. Phys. Chem. Lett. 2015, 6, 1435.

[24] Hu, J.; Wang, L.; Shi, L.; Huang, H. J. Power Sources 2014, 269, 144.

[25] Liu, Y.; Dai, H.; Du, Y.; Deng, J.; Zhang, L.; Zhao, Z.; Au, C. T. J. Catal. 2012, 287, 149 .

[26] Lee, Y. C.; Peng, P. Y.; Chang, W. S.; Huang, C. M. J. Taiwan Chem. Eng. 2014, 45, 2334.

[27] Stöber, W.; Fink, A.; Bohn, E. J. Colloid Interface Sci. 1968, 26, 62.

[28] Yang, C. H.; Chen, B. J.; Lu, J.; Yang, J. H.; Zhou, J.; Chen, Y. M.; Suo, Z. Extreme Mech. Lett. 2015, 25, 59.

[29] Tan, P.; Chen, B.; Xu, H.; Zhang, H.; Cai, W.; Ni, M.; Liu, M.; Shao, Z. Energy Environ. Sci. 2017, 10, 2056.

[30] Song, S.-D.; Tang, Z.-Y.; Pan, L.-Z.; Nan, J.-M. Acta Chim. Sinica 2005，63，363. (宋世栋，唐致远，潘丽珠，南俊民，化学学报, 2005, 63, 363.)

[31] Liu, Y.; Li, J.; Li, W.; Li, Y.; Zhan, F.; Tang, H.; Chen, Q. Int. J. Hydrogen Energy 2016, 41, 10354.

[32] Zhang, Z.; Zuo, C.; Liu, Z.; Yu, Y.; Zuo, Y.; Song, Y. J. Power Sources 2014, 251, 470 .

(Zhao, C.) 